\title{
Topologically Protected Landau Level in the Vortex Lattice of a Weyl Superconductor
}

\author{
M. J. Pacholski, ${ }^{1}$ C. W. J. Beenakker, ${ }^{1}$ and İ. Adagideli ${ }^{2}$ \\ ${ }^{1}$ Instituut-Lorentz, Universiteit Leiden, P.O. Box 9506, 2300 RA Leiden, The Netherlands \\ ${ }^{2}$ Faculty of Engineering and Natural Sciences, Sabanci University, Orhanli-Tuzla, Istanbul, Turkey
}

(Received 4 October 2017; published 16 July 2018)

\begin{abstract}
The question whether the mixed phase of a gapless superconductor can support a Landau level is a celebrated problem in the context of $d$-wave superconductivity, with a negative answer: the scattering of the subgap excitations (massless Dirac fermions) by the vortex lattice obscures the Landau level quantization. Here we show that the same question has a positive answer for a Weyl superconductor: the chirality of the Weyl fermions protects the zeroth Landau level by means of a topological index theorem. As a result, the heat conductance parallel to the magnetic field has the universal value $G=\frac{1}{2} g_{0} \Phi / \Phi_{0}$, with $\Phi$ as the magnetic flux through the system, $\Phi_{0}$ as the superconducting flux quantum, and $g_{0}$ as the thermal conductance quantum.
\end{abstract}

DOI: 10.1103/PhysRevLett.121.037701

Introduction.-In 1998, Gor'kov and Schrieffer [1] and Anderson [2] made the remarkable prediction that the excitation spectrum in the mixed phase of a high- $T_{c}$ superconductor (with massless quasiparticles at nodal points of the $d$-wave pair potential) has the Landau levels of the relativistic Dirac equation. This was nearly a decade before the quantum Hall effect of massless electrons was measured in graphene [3,4], and it would have marked the first appearance in the solid state of a magnetic-field independent zeroth Landau level.

It did not turn out that way: the spatially varying supercurrent in the Abrikosov vortex lattice strongly scatters the quasiparticles [5], even if the vortices overlap and produce a uniform magnetic field. Since Franz and Tešanović [6], we know that the quasiparticles in the mixed phase of a $d$-wave superconductor retain the zero-field Dirac cone, the main effect of the magnetic field being a renormalization of the Fermi velocity [7-16]. Recent proposals [17-19] use strain to mimic the effect of a magnetic field in a $d$-wave superconductor without breaking time-reversal symmetry, but the coexistence of Landau levels and a vortex lattice has remained elusive.

Here we propose that Weyl superconductors can make it happen. A Weyl semimetal with induced $s$-wave superconductivity has massless nodal quasiparticles in a 3D Weyl cone [20,21], with the same linear dispersion as the 2D Dirac cone of a $d$-wave superconductor [22,23]. We compare the band structures in Fig. 1 (see Supplemental Material [24] for a detailed calculation), where the momentum follows a path through the magnetic Brillouin zone of Fig. 2. In zero magnetic field, the gapless nodal points at the Fermi level $(E=0)$ are qualitatively the same in both superconductors. But the response to a vortex lattice is fundamentally different: while in the $d$-wave superconductor, the dispersive Dirac cones persist, as expected [6], in the Weyl superconductor, a zeroth Landau level appears that is completely dispersionless in the plane perpendicular to the magnetic field.

We will return to these numerical calculations later on, but first we want to explain why the zeroth Landau level in a Weyl superconductor is not broadened by the vortex lattice, as it is in a $d$-wave superconductor. We have traced the origin of the difference to the topological protection of the zero mode enforced by an index theorem for Hamiltonians with chiral symmetry [34]. For this explanation, we will use an effective low-energy Hamiltonian. The numerics uses the full Hamiltonian and serves as a test of our analytics. We conclude with a discussion of the universal thermal conductance supported by the zero mode.



FIG. 1. Excitation spectrum of a nodal superconductor in zero magnetic field (black dashed curves) and in the mixed phase with a square lattice of Abrikosov vortices (red solid curves) [33]. (a) 2D $d$-wave superconductor. (b) 3D Weyl superconductor (with $k_{z}=\pi / 3$ at the Weyl point). The momentum follows a path through the magnetic Brillouin zone of Fig. 2. The location of the zero-field Dirac and Weyl points is indicated by green arrows. The $n$th Landau level is expected at $E_{n}=\sqrt{n} E_{1}$, with $E_{1}=2 \sqrt{\pi} v_{F} / d_{0}$. In the $d$-wave superconductor, the Landau levels are destroyed by the vortex lattice [6], while in the Weyl superconductor, they are protected by chiral symmetry. 


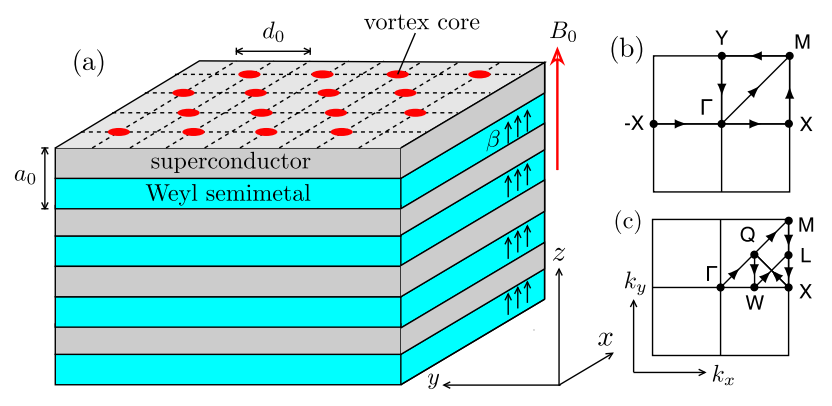

FIG. 2. Weyl superconductor in the mixed phase. (a) A Weyl semimetal-superconductor heterostructure (layers of a topological insulator, with perpendicular magnetization $\beta$, separated by $s$-wave superconducting spacer layers [20]). A magnetic field $B_{0}$ is applied perpendicular to the layers. The heterostructure has lattice constant $a_{0}$, while the square vortex array has lattice constant $d_{0}$ (with two $h / 2 e$ vortices per unit cell). (b),(c) Two different paths through the magnetic Brillouin zone of the vortex array.

Weyl superconductor in the mixed phase.-We start quite generally from the Bogoliubov-De Gennes (BdG) Hamiltonian in the Anderson gauge [2],

$$
\begin{aligned}
\mathcal{H}(\boldsymbol{k}) & =U^{\dagger}\left(\begin{array}{cc}
H_{0}(\boldsymbol{k}-e \boldsymbol{A}) & \Delta \\
\Delta^{*} & -\sigma_{y} H_{0}^{*}(-\boldsymbol{k}-e \boldsymbol{A}) \sigma_{y}
\end{array}\right) U \\
& =\left(\begin{array}{cc}
H_{0}\left(\boldsymbol{k}+\boldsymbol{a}+m \boldsymbol{v}_{s}\right) & \Delta_{0} \\
\Delta_{0} & -\sigma_{y} H_{0}^{*}\left(-\boldsymbol{k}-\boldsymbol{a}+m \boldsymbol{v}_{s}\right) \sigma_{y}
\end{array}\right),
\end{aligned}
$$

with the definitions $(\hbar \equiv 1$, electron charge $+e$, mass $m)$ $U=\left(\begin{array}{cc}e^{i \phi} & 0 \\ 0 & 1\end{array}\right), \quad \boldsymbol{a}=\frac{1}{2} \nabla \phi, \quad m v_{s}=\frac{1}{2} \nabla \phi-e \boldsymbol{A}$.

The $2 \times 2$ matrix structure of $H$ refers to electron and hole quasiparticles, with single-particle Hamiltonian $H_{0}$ and its time reverse in the diagonal blocks, coupled by the superconducting pair potential $\Delta=\Delta_{0} e^{i \phi}$ in the off-diagonal blocks. The unitary transformation $U$ removes the spatially dependent phase $\phi(x, y)$ from the pair potential into the single-particle Hamiltonian, where it combines with the vector potential $\boldsymbol{A}(x, y)$ in the $x-y$ plane, corresponding to the magnetic field $\boldsymbol{B}=\nabla \times \boldsymbol{A}$ along $z$.

Both the gauge field $\boldsymbol{a}(x, y)$ and the supercurrent velocity $\boldsymbol{v}_{s}(x, y)$ wind around the positions $\boldsymbol{R}_{n}$ of the vortex cores, according to

$$
\nabla \times \nabla \phi=2 \pi \hat{z} \sum_{n} \delta\left(\boldsymbol{r}-\boldsymbol{R}_{n}\right) .
$$

(For definiteness, we assume the field points in the positive $z$ direction.) A spatial average over the vortices gives a vanishing supercurrent velocity, $\overline{\boldsymbol{v}}_{s}=0$, while the average $\overline{\nabla \times \boldsymbol{a}}=e \overline{\boldsymbol{B}}$ gives the average magnetic field. The field is approximately uniform, equal to $B_{0}$, in the mixed phase
$H_{c 1} \ll B_{0} \ll H_{c 2}$ of a type-II superconductor with overlapping vortices. In this regime, the vortex cores occupy only a small fraction $B_{0} / H_{c 2} \ll 1$ of the volume, so the amplitude $\Delta_{0}$ of the pair potential is also approximately uniform and only the phase $\phi$ is strongly position dependent.

We now specify to a Weyl superconductor, in the heterostructure configuration of Meng and Balents [20,35]: a stack in the $z$ direction of layers of Weyl semimetal alternating with an $s$-wave superconductor. A magnetization $\beta$ perpendicular to the layers separates the Weyl cones in the Brillouin zone along $k_{z}$. The Weyl points are at $\boldsymbol{k}=(0,0, \pm K), v_{F}^{2} K^{2}=$ $\beta^{2}-\Delta_{0}^{2}$, with $v_{F}$ the Fermi velocity (assumed isotropic for simplicity). The Weyl cones remain gapless as long as $\Delta_{0}<\beta$ [37].

In the BdG Hamiltonian (1) each Weyl cone is doubled into an electron and hole cone, mixed by the pair potential. We describe this mixing following Ref. [38], in the simplest case that the Weyl cones are close to the center $\boldsymbol{k}=0$ of the Brillouin zone and we may linearize the momenta. (All nonlinearities in the full Brillouin zone are included in our numerics.) The single-particle Weyl Hamiltonian $H_{0}$ is a $4 \times 4$ matrix,

$$
H_{0}(\boldsymbol{k})=v_{F} \tau_{z} \boldsymbol{k} \cdot \boldsymbol{\sigma}+\beta \tau_{0} \sigma_{z}-\mu \tau_{0} \sigma_{0},
$$

with $\mu$ as the chemical potential. It is composed from Pauli matrices $\sigma_{\alpha}$ and $\tau_{\alpha}$ that act on the spin and orbital degree of freedom, respectively. We also need a third set of Pauli matrices $\nu_{\alpha}$ in the electron-hole basis. (The corresponding $2 \times 2$ unit matrices are $\sigma_{0}, \tau_{0}, \nu_{0}$.)

A unitary transformation $\mathcal{H} \mapsto V^{\dagger} \mathcal{H} V$ with

$V=\exp \left(\frac{1}{2} i \theta \nu_{y} \tau_{z} \sigma_{z}\right), \quad \tan \theta=-\frac{\Delta_{0}}{v_{F} k_{z}}, \quad \theta \in(0, \pi)$,

followed by a projection onto the $\nu=\tau= \pm 1$ blocks, gives for the Weyl cones an effective $2 \times 2$ low-energy Hamiltonian [39]

$$
\begin{gathered}
H_{ \pm}(\boldsymbol{k})=v_{F} \sum_{\alpha=x, y}\left(k_{\alpha}+a_{\alpha} \pm \kappa m v_{s, \alpha}\right) \sigma_{\alpha} \\
+\left(\beta-m_{k_{z}}\right) \sigma_{z} \mp \kappa \mu \sigma_{0}, \\
m_{k_{z}}=\sqrt{\Delta_{0}^{2}+v_{F}^{2} k_{z}^{2}}, \quad \kappa=-v_{F} k_{z} / m_{k_{z}} .
\end{gathered}
$$

The electron- and hole-like cones have the opposite sign of the effective charge $q_{\text {eff }}= \pm \kappa e$, with $\left|q_{\text {eff }}\right| \rightarrow$ $e \sqrt{1-\Delta_{0}^{2} / \beta^{2}}$ for $\left|k_{z}\right| \rightarrow K$, smaller than the bare charge $e$ due to the mixing of electrons and holes by the pair potential [40]. The velocity $v_{z}=\partial m_{k_{z}} / \partial k_{z}$ perpendicular to the layers is also renormalized by the superconductivity: $v_{z} \rightarrow v_{F}^{2} K / \beta$ for $\left|k_{z}\right| \rightarrow K$.

At the Weyl point, for $\mu=0$ and $\left|k_{z}\right|=K$, the Hamiltonian (6) anticommutes with $\sigma_{z}$. This so-called chiral symmetry gives a formal correspondence with a 
problem first studied 40 years ago by Aharonov and Casher [41], as an application of an index theorem from supersymmetric quantum mechanics [34]. The problem of Ref. [41], to determine the zeroth Landau level of a two-dimensional massless electron in an inhomogeneous magnetic field, has also been studied more recently in the context of graphene [42-44]. We need to adapt the calculation here to account for the fractionally charged quasiparticles, but the basic approach carries through.

Calculation of the zero modes.-To study the effect of chiral symmetry on the Landau level spectrum, we set $\mu=0,\left|k_{z}\right|=K$, and focus our attention on the chiral Hamiltonian

$$
\begin{aligned}
H_{\text {chiral }} & =v_{F}\left(\begin{array}{cc}
0 & \mathcal{D} \\
\mathcal{D}^{\dagger} & 0
\end{array}\right), \quad \mathcal{D}=\Pi_{x}-i \Pi_{y}, \\
\boldsymbol{\Pi} & =-i \nabla+e \mathcal{A}, \quad e \mathcal{A}=\boldsymbol{a} \pm \kappa m \boldsymbol{v}_{s} .
\end{aligned}
$$

(We omit the \pm subscript for ease of notation.) The effective vector potential $\mathcal{A}$ describes the effective magnetic field

$\mathcal{B}=\partial_{x} \mathcal{A}_{y}-\partial_{y} \mathcal{A}_{x}=\Phi_{0}(1 \pm \kappa) \sum_{n} \delta\left(\boldsymbol{r}-\boldsymbol{R}_{n}\right) \mp \kappa B$

felt by the Weyl fermions in the vortex lattice.

For what follows, it is convenient to choose a gauge such that $\nabla \cdot \mathcal{A}=0$ and to assume that the external magnetic field $B_{0}$ is imposed on a large but finite area $S$. Because there are $N_{\text {vortex }}=B_{0} S / \Phi_{0}$ vortices in that area (with $\Phi_{0}=h / 2 e$ the superconducting flux quantum), the flux $\Phi=\int d \boldsymbol{r} \mathcal{B}=B_{0} S$ through the system corresponding to the effective field equals the real flux. (The $\kappa$ dependence of $\mathcal{B}$ drops out upon spatial integration.)

A zero mode $\psi$ of $H_{\text {chiral }}$ is either a spinor $\left(\begin{array}{l}u \\ 0\end{array}\right)$ with $\mathcal{D}^{\dagger} u=$ 0 or it is a spinor $\left(\begin{array}{l}0 \\ v\end{array}\right)$ with $\mathcal{D} v=0$. The general solution of these two differential equations has the form $[41,43,45]$

$$
\begin{aligned}
u & =f(\zeta) e^{W}, \quad v=f\left(\zeta^{*}\right) e^{-W}, \quad \zeta=x+i y, \\
W(\boldsymbol{r}) & =\frac{1}{2 \Phi_{0}} \int d x^{\prime} \int d y^{\prime} \mathcal{B}\left(\boldsymbol{r}^{\prime}\right) \ln \left|\boldsymbol{r}-\boldsymbol{r}^{\prime}\right| .
\end{aligned}
$$

The difference $\mathcal{N}=N_{u}-N_{v}$ in the number of normalizable solutions for $u$ and $v$ is called the index of $H_{\text {chiral }}$. The absolute value $|\mathcal{N}|$ is a lower bound on the degeneracy of the zero mode and the sign of $\mathcal{N}$ determines the chirality: whether the zero mode is an eigenstate of $\sigma_{z}$ with eigenvalue +1 or -1 .

To determine the index of $H_{\text {chiral }}$, we proceed as follows. In the absence of vortices, the function $f(\zeta)$ is analytic in the entire complex plane and we can use a basis of polynomials. A polynomial $f(\zeta)$ of degree $N-1$ then produces $N$ linearly independent zero modes-provided $u$ or $v$ is normalizable, $\int r d r|\psi|^{2}<\infty$. For large $r$, one has asymptotically

$$
W \rightarrow \frac{1}{2}\left(\Phi / \Phi_{0}\right) \ln |\boldsymbol{r}| \Rightarrow e^{W} \rightarrow|\boldsymbol{r}|^{N_{\text {vortex }} / 2},
$$

so, if only the decay at infinity would be an issue, we would conclude that $N_{u}=0, N_{v}=\operatorname{Int}\left[N_{\text {vortex }} / 2\right]$. This is the answer in the absence of vortices [41], when the degeneracy of the zero mode is determined by the enclosed flux in units of $h / e=2 \Phi_{0}$, while the chirality is set by the sign of the magnetic field (which we have assumed positive). As we will now show, the presence of vortices introduces a dependence of the chirality on the sign of the fractional charge $q_{\text {eff }}= \pm \kappa e$ of the quasiparticles, while the degeneracy remains given by the bare electron charge $e$.

With vortices, the function $f(\zeta)$ may have poles at the vortex cores $\zeta_{n}=x_{n}+i y_{n}$. We use this freedom to reexpress the solution (10) as

$$
u=g(\zeta) e^{W} \prod_{n}\left(\zeta-\zeta_{n}\right)^{-1}, \quad v=f\left(\zeta^{*}\right) e^{-W} .
$$

If for $f$ and $g$ we take polynomials of degree $N-1$, with $N=\operatorname{Int}\left[N_{\text {vortex }} / 2\right]$, then both the functions $u$ and $v$ decay sufficiently rapidly at infinity. The boundary condition at the vortex cores now determines which of the two solutions is realized.

Near a vortex at position $\boldsymbol{r}_{n}$ the asymptotics is

$|u|^{2} \rightarrow\left|\boldsymbol{r}-\boldsymbol{r}_{n}\right|^{-1+q_{\mathrm{eff}} / e}, \quad|v|^{2} \rightarrow\left|\boldsymbol{r}-\boldsymbol{r}_{n}\right|^{-1-q_{\mathrm{eff}} / e}$.

Since $\left|q_{\text {eff }}\right|<e$ both solutions $\psi_{u}=\left(\begin{array}{c}u \\ 0\end{array}\right)$ and $\psi_{v}=\left(\begin{array}{l}0 \\ v\end{array}\right)$ remain square integrable at the vortex core. The boundary condition [24]

$$
\sigma_{z} \psi=\left(\operatorname{sgn} q_{\mathrm{eff}}\right) \psi, \quad \text { for } \boldsymbol{r} \rightarrow \boldsymbol{r}_{n}
$$

selects the most weakly divergent solution in Eq. (13): $\psi=\psi_{u}$ with positive chirality for $q_{\text {eff }}>0$ and $\psi=\psi_{v}$ with negative chirality for $q_{\text {eff }}<0$.

All of this was for $\mu=0,\left|k_{z}\right|=K$, but both terms $\mu \sigma_{0}$ and $\left(\beta-m_{k_{z}}\right) \sigma_{z}$ from Eq. (6) can be immediately reinstated since the zero mode is an eigenstate of $\sigma_{z}$. The resulting $\mu$ and $k_{z}$ dependence of the zeroth Landau level is

$$
E_{ \pm}\left(k_{z}\right)=\mp \kappa \mu+\left(\operatorname{sgn} q_{\mathrm{eff}}\right)\left(\beta-m_{k_{z}}\right) .
$$

We have thus seen how the chiral symmetry protects the zeroth Landau level from being destroyed by the vortex lattice. To complete this analytical treatment, we point out why the $d$-wave superconductor lacks a similar protection. In the Anderson gauge, the low-energy Hamiltonian near the nodal point of a $d$-wave pair potential reads $[2,6,11]$

$H_{d \text {-wave }}=v_{F}\left(k_{x}+a_{x}\right) \sigma_{z}+v_{\Delta}\left(k_{y}+a_{y}\right) \sigma_{x}+m v_{s, x} \sigma_{0}$.

There are inessential differences with $H_{\text {chiral }}$ from Eq. (8) the Dirac cone is anisotropic and the basis of Pauli matrices is rotated - but the essential difference is that the superfluid 


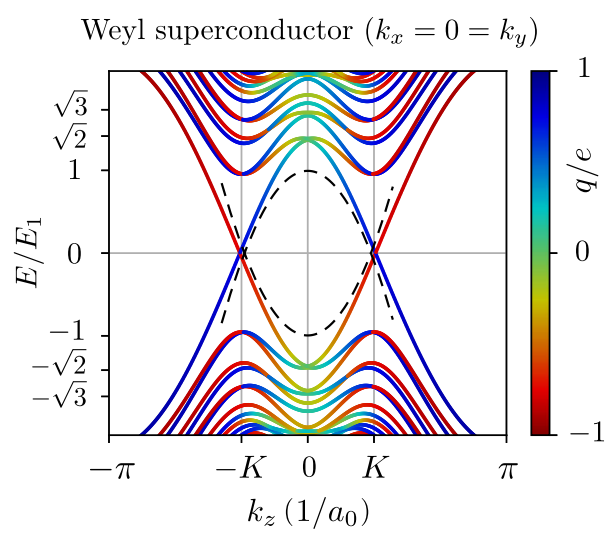

FIG. 3. Same as Fig. 1(b), but now as a function of $k_{z}$ for $k_{x}=0=k_{y}$ at the center of the Brillouin zone [47]. The color scale indicates the charge expectation value. The dashed curve is the dispersion (15) of the zeroth Landau level, calculated analytically for $K \ll 1$ (which explains the deviation from the numerics). The effective charge at $E=0$ is \pm 0.73 , close to the analytical prediction of $\pm \kappa= \pm 1 / \sqrt{2}$.

velocity breaks the chiral symmetry: $H_{d \text {-wave }}$ anticommutes with $\sigma_{y}$ only if $v_{s, x}=0$. In the $d$-wave superconductor, the superfluid velocity enters as a chirality-breaking scalar potential, while in the Weyl superconductor, it is a chiralitypreserving vector potential. The former is a strong scatterer, which effectively destroys the Landau levels, while the latter cannot by force of the topological index theorem.

Comparison with numerics.-To test our analytical theory, we have numerically calculated the spectrum of a Weyl superconductor with a vortex lattice, using the KWANT tight-binding code [46]. The $8 \times 8$ Hamiltonian has the BdG form (1) with [20,21,36]

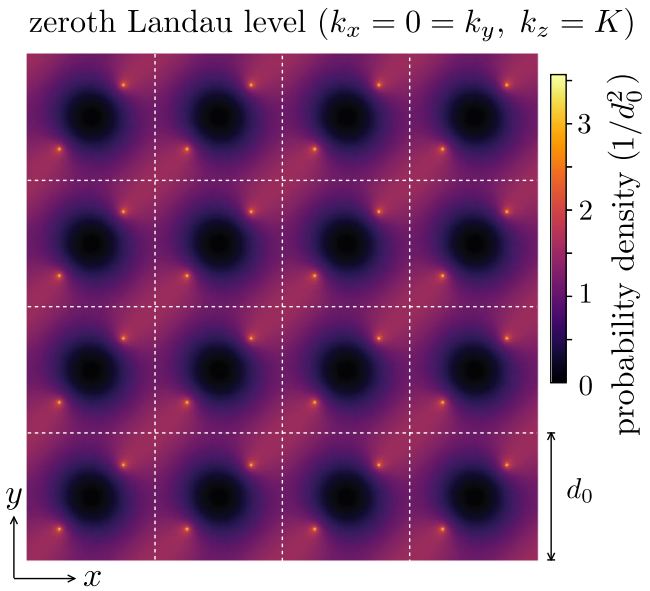

FIG. 4. Color scale plot of $|\psi(x, y)|^{2}$ in the zeroth Landau level of the Weyl superconductor [48]. The white dashed lines indicate the vortex array, with a pair of $h / 2 e$ vortices in each unit cell. On approaching a vortex core, when the separation $\delta r \rightarrow 0$, the density diverges as a power law $|\psi|^{2} \propto \delta r^{1 / \sqrt{2}-1}$, in accord with Eq. (13).

$$
\begin{aligned}
H_{0}(\boldsymbol{k})= & t_{0} \sum_{\alpha=x, y, z}\left[\tau_{z} \sigma_{\alpha} \sin k_{\alpha} a_{0}+\tau_{x} \sigma_{0}\left(1-\cos k_{\alpha} a_{0}\right)\right] \\
& +\beta \tau_{0} \sigma_{z}-\mu \tau_{0} \sigma_{0} .
\end{aligned}
$$

Near the center of the Brillouin zone, this reduces to the linearized Hamiltonian (4), but now we will not make any linearization. Results are shown in Figs. 1(b), 3, and 4 (see Supplemental Material [24]). They are fully consistent with the analytics.

Thermal conductance.-The chiral zeroth Landau level governs the thermal transport properties of the Weyl superconductor, in the direction parallel to the magnetic field. The degeneracy $e B_{0} S / h=\frac{1}{2} \Phi / \Phi_{0}$ of the zeroth Landau level implies a thermal conductance

$$
G=\frac{1}{2} g_{0} \Phi / \Phi_{0}, g_{0}=\mathcal{L} T e^{2} / h,
$$

with $\mathcal{L}=\frac{1}{3}\left(\pi k_{B} / e\right)^{2}$ as the Lorenz number. In other words, each vortex contributes half a thermal conductance quantum to the heat transport- the factor $1 / 2$ being a reminder that the quasiparticles in the Weyl superconductor are Majorana fermions [40]. Do note that the states in the zeroth Landau level are extended over the $x-y$ plane, the current flow is not confined to the vortex cores (see Fig. 4) [49]. We expect the universal thermal conductance (18) to be robust against nonmagnetic disorder, which in the effective Hamiltonian would enter as a term $\propto \sigma_{z}$ that does not couple Landau levels of opposite chirality.

Conclusion.-In this Letter, we have revisited the celebrated question [1,2] of whether quasiparticles in the vortex lattice of a gapless superconductor can condense into Landau levels. We have shown that Weyl superconductors can accomplish what $d$-wave superconductors could not [6]: the chirality of Weyl fermions protects the zeroth Landau level from broadening due to scattering by the vortices. We have developed the analytical argument for a simple low-energy Hamiltonian and supported it by numerical calculations for a heterostructure model of the Weyl superconductor [20]. We anticipate that the Landau levels will govern the thermodynamic and transport properties of the vortex lattice, finally allowing for the observation of quantum effects that proved elusive in the $d$-wave context.

We have benefited from discussions with D. I. Pikulin and J. Tworzydło. This research was supported by the Netherlands Organization for Scientific Research (NWO/ OCW), an ERC Synergy Grant, and by the TÜBİTAK Grant No. 114F163.

[1] L. P. Gor'kov and J. R. Schrieffer, De Haas-van Alphen Effect in Anisotropic Superconductors in Magnetic Fields Well below $H_{c 2}$, Phys. Rev. Lett. 80, 3360 (1998). 
[2] P. W. Anderson, Anomalous magnetothermal resistance of high- $T_{c}$ superconductors: Anomalous cyclotron orbits at a Dirac point, arXiv:cond-mat/9812063.

[3] K. S. Novoselov, A. K. Geim, S. V. Morozov, D. Jiang, M. I. Katsnelson, I. V. Grigorieva, S. V. Dubonos, and A. A. Firsov, Two-dimensional gas of massless Dirac fermions in graphene, Nature (London) 438, 197 (2005).

[4] Y. Zhang, Y.-W. Tan, H. L. Stormer, and P. Kim, Experimental observation of the quantum Hall effect and Berry's phase in graphene, Nature (London) 438, 201 (2005).

[5] A. S. Mel'nikov, Quantization of the quasiparticle spectrum in the mixed state of d-wave superconductors, J. Phys. Condens. Matter 11, 4219 (1999).

[6] M. Franz and Z. Tešanović, Quasiparticles in the Vortex Lattice of Unconventional Superconductors: Bloch Waves or Landau Levels?, Phys. Rev. Lett. 84, 554 (2000).

[7] K. Yasui and T. Kita, Quasiparticles of $d$-Wave Superconductors in Finite Magnetic Fields, Phys. Rev. Lett. 83, 4168 (1999).

[8] L. Marinelli, B. I. Halperin, and S. H. Simon, Quasiparticle spectrum of $d$-wave superconductors in the mixed state, Phys. Rev. B 62, 3488 (2000).

[9] N. B. Kopnin and V. M. Vinokur, Magnetic quantization of electronic states in $d$-wave superconductors, Phys. Rev. B 62, 9770 (2000).

[10] Y. Morita and Y. Hatsugai, Duality in the Azbel-Hofstadter Problem and Two-Dimensional $d$-Wave Superconductivity with a Magnetic Duality, Phys. Rev. Lett. 86, 151 (2001).

[11] O. Vafek, A. Melikyan, M. Franz, and Z. Tešanović, Quasiparticles and vortices in unconventional superconductors, Phys. Rev. B 63, 134509 (2001).

[12] D. Knapp, C. Kallin, and A. J. Berlinsky, Dirac quasiparticles in the mixed state, Phys. Rev. B 64, 014502 (2001).

[13] O. Vafek, A. Melikyan, and Z. Tešanović, Quasiparticle Hall transport of $d$-wave superconductors in the vortex state, Phys. Rev. B 64, 224508 (2001).

[14] A. Vishwanath, Quantized Thermal Hall Effect in the Mixed State of $d$-Wave Superconductors, Phys. Rev. Lett. 87, 217004 (2001)

[15] O. Vafek and A. Melikyan, Index Theoretic Characterization of $d$-Wave Superconductors in the Vortex State, Phys. Rev. Lett. 96, 167005 (2006).

[16] A. Melikyan and Z. Tešanović, Dirac-Bogoliubov-de Gennes quasiparticles in a vortex lattice, Phys. Rev. B 76, 094509 (2007).

[17] G. Massarelli, G. Wachtel, J. Y. T. Wei, and A. Paramekanti, Pseudo-Landau levels of Bogoliubov quasiparticles in nodal superconductors, Phys. Rev. B 96, 224516 (2017).

[18] E. M. Nica and M. Franz, Landau levels from neutral Bogoliubov particles in two-dimensional nodal superconductors under strain and doping gradients, Phys. Rev. B 97, 024520 (2018).

[19] T. Liu, M. Franz, and S. Fujimoto, Quantum oscillations and Dirac-Landau levels in Weyl superconductors, Phys. Rev. B 96, 224518 (2017); M. Franz Appendix D of their paper contains a numerical confirmation of our Fig. 1(b), added in response to our preprint (private communication).

[20] T. Meng and L. Balents, Weyl superconductors, Phys. Rev. B 86, 054504 (2012); Erratum, Phys. Rev. B 96, 019901(E) (2017).
[21] G. Bednik, A. A. Zyuzin, and A. A. Burkov, Superconductivity in Weyl metals, Phys. Rev. B 92, 035153 (2015).

[22] G. E. Volovik, Superconductivity with lines of gap nodes: Density of states in the vortex, JETP Lett. 58, 469 (1993). http://www.jetpletters.ac.ru/ps/1189/article_17954.shtml.

[23] S. H. Simon and P. A. Lee, Scaling of the Quasiparticle Spectrum for $d$-Wave Superconductors, Phys. Rev. Lett. 78, 1548 (1997).

[24] See the Supplemental Material at http://link.aps.org/ supplemental/10.1103/PhysRevLett.121.037701, which includes Refs. [25-32], for (A) details regarding the derivation of the boundary condition at the vortex core, (B) details of the tight-binding calculation, and (C) demonstration of the power law scaling of the quasiparticle density near the vortex core. The appendices also include a demonstration of the robustness of our results to (D) anisotropic Weyl cones and arbitrary orientation of the magnetic field and (E) tilting of the Weyl cones, all the way up to the type-I-II transition.

[25] A. De Martino and R. Egger, On the spectrum of a magnetic quantum dot in graphene, Semicond. Sci. Technol. 25, 034006 (2010).

[26] E. Brown, Bloch electrons in a uniform magnetic field, Phys. Rev. 133, A1038 (1964).

[27] J. Zak, Magnetic translation group, Phys. Rev. 134, A1602 (1964); Magnetic translation group. II. Irreducible representations, Phys. Rev. 134, A1607 (1964).

[28] H. J. Fischbeck, Theory of Bloch electrons in a magnetic field, Phys. Status Solidi 38, 11 (1970).

[29] M. Diez, I. C. Fulga, D. I. Pikulin, M. Wimmer, A. R. Akhmerov, and C. W. J. Beenakker, Phase-locked magnetoconductance oscillations as a probe of Majorana edge states, Phys. Rev. B 87, 125406 (2013).

[30] A. A. Soluyanov, D. Gresch, Z. Wang, Q. Wu, M. Troyer, X. Dai, and B. A. Bernevig, Type-II Weyl semimetals, Nature (London) 527, 495 (2015).

[31] T. Kawarabayashi, Y. Hatsugai, T. Morimoto, and H. Aoki, Generalized chiral symmetry and stability of zero modes for tilted Dirac cones, Phys. Rev. B 83, 153414 (2011).

[32] T. Kawarabayashi, Y. Hatsugai, T. Morimoto, and H. Aoki, Generalization of chiral symmetry for tilted Dirac cones, Int. J. Mod. Phys. Conf. Ser. 11, 145 (2012).

[33] In dimensionless units $\left(t_{0}, a_{0}, \hbar \equiv 1\right)$, the parameters for the band structure of Fig. 1(a) are $\Delta_{0}=1\left(d_{x^{2}-y^{2}}\right.$ pairing), $v_{F}=$ $v_{\Delta}=2 \sqrt{2}$ (isotropic Dirac cone), $\mu=4$ (band center), $d_{0}=49, E_{1}=4 \sqrt{2 \pi} / d_{0}$; for Fig. 1(b) they are $\Delta_{0}=1$ ( $s$-wave pairing), $\beta=\sqrt{2}, v_{F}=1$ (isotropic Weyl cone), $\mu=0$ (Weyl point), $d_{0}=49, E_{1}=2 \sqrt{\pi} / d_{0}$. In the Weyl semimetal with a vortex lattice, we increased $\mu$ slightly from 0 to 0.0031 to line up with the crossing point of the zeroth Landau levels of opposite chirality.

[34] L. Alvarez-Gaumé, Supersymmetry and the Atiyah-Singer index theorem, Commun. Math. Phys. 90, 161 (1983).

[35] We use the heterostructure model of Ref. [20] for concreteness, but we have checked that the Landau levels appear as well in the model of Ref. [21], which refers to a Weyl semimetal with intrinsic superconductivity. The difference between the two models, scalar versus pseudoscalar pairing [36], does not affect the topological protection.

[36] N. Bovenzi, M. Breitkreiz, P. Baireuther, T. E. O’Brien, J. Tworzydło, I. Adagideli, and C. W. J. Beenakker, Chirality 
blockade of Andreev reflection in a magnetic Weyl semimetal, Phys. Rev. B 96, 035437 (2017).

[37] A supercurrent perpendicular to the layers can gap out the Weyl cones even if $\Delta_{0}<\beta$, but for now we only consider supercurrents flowing in the plane of the layers. In the presence of time-reversal symmetry, for $\beta=0$, the Weyl superconductor is gapped except at the vortex cores, so no Landau level can exist in the bulk.

[38] T. E. O'Brien, C. W. J. Beenakker, and İ. Adagideli, Superconductivity Provides Access to the Chiral Magnetic Effect of an Unpaired Weyl Cone, Phys. Rev. Lett. 118, 207701 (2017).

[39] The low-energy Hamiltonian (6) does not include virtual transitions to higher bands, of second order in $\mu$ and $v_{s}$. These are included in the numerics, which is based on the full Hamiltonian.

[40] P. Baireuther, J. Tworzydło, M. Breitkreiz, İ. Adagideli, and C. W. J. Beenakker, Weyl-Majorana solenoid, New J. Phys. 19, 025006 (2017).

[41] Y. Aharonov and A. Casher, Ground state of a spin-1/2 charged particle in a two-dimensional magnetic field, Phys. Rev. A 19, 2461 (1979).

[42] M. I. Katsnelson and M. F. Prokhorova, Zero-energy states in corrugated bilayer graphene, Phys. Rev. B 77, 205424 (2008).

[43] J. Kailasvuori, Pedestrian index theorem à la AharonovCasher for bulk threshold modes in corrugated multilayer graphene, Europhys. Lett. 87, 47008 (2009).
[44] C.-K. Lu and B. Seradjeh, Zero modes of the generalized fermion-vortex system in magnetic field, Phys. Rev. B 89, 245448 (2014).

[45] To verify Eq. (10), first note that $\left(-i \partial_{x} \pm \partial_{y}\right) f(x \pm i y)=0$, so we only need to consider derivatives of $W$. For that purpose, it is helpful to write $\mathcal{A}=\nabla \times \hat{z} \omega$ (which is possible in the gauge where $\nabla \cdot \mathcal{A}=0$ ), then note that $\mathcal{B}=-\nabla^{2} \omega$ and use the identity $\nabla^{2} \ln \left|\boldsymbol{r}-\boldsymbol{r}^{\prime}\right|=2 \pi \delta\left(\boldsymbol{r}-\boldsymbol{r}^{\prime}\right)$, to derive that $W=$ $-\left(\pi / \Phi_{0}\right) \omega=-e \omega$ and $\left(-i \partial_{x} \pm \partial_{y}\right) W=\mp e \mathcal{A}_{x}-i e \mathcal{A}_{y}$. Hence, one concludes that $\left(\Pi_{x} \pm i \Pi_{y}\right) e^{ \pm W}=0$.

[46] C. W. Groth, M. Wimmer, A. R. Akhmerov, and X. Waintal, KWANT: A software package for quantum transport, New J. Phys. 16, 063065 (2014).

[47] The parameters for the band structure of Fig. 3 are the same as those of Fig. 1(b), except that we took a larger magnetic field $\left(d_{0}=10\right)$ so that the Landau level splitting is more clearly visible on this scale.

[48] The wave function in Fig. 4 is evaluated for the same parameters as Fig. 1(b), but at a smaller magnetic field $\left(d_{0}=202\right)$, to have a smaller overlap of the vortices. There are two zero modes of opposite effective charge $q_{\text {eff }}= \pm \kappa e$, with identical density profile so we only show one of them.

[49] As we will discuss elsewhere, it is possible to concentrate the heat flow to the vortex cores by applying a flux bias, and in that way realize a situation reminiscent of the axion insulator of T. Schuster, T. Iadecola, C. Chamon, R. Jackiw, and S.-Y. Pi, Dissipationless conductance in a topological coaxial cable, Phys. Rev. B 94, 115110 (2016). 